\title{
Aymon de Montfalcon et l'Observance : la fondation controversée des couvents de Savigny, de Sainte-Catherine du Jorat et de Morges
}

\section{Stéphanie Vocanson-Manzi}

\section{CpenEdition}

Journals

Édition électronique

URL : http://journals.openedition.org/edl/1318

DOI : $10.4000 /$ edl. 1318

ISSN : 2296-5084

Éditeur

Université de Lausanne

\section{Édition imprimée}

Date de publication : 1 décembre 2018

Pagination : 47-62

ISBN : 978-2-940331-69-7

ISSN : 0014-2026

\section{Référence électronique}

Stéphanie Vocanson-Manzi, « Aymon de Montfalcon et l'Observance : la fondation controversée des couvents de Savigny, de Sainte-Catherine du Jorat et de Morges », Études de lettres [En ligne], 3-4 I 2018, mis en ligne le 15 décembre 2020, consulté le 17 décembre 2020. URL : http:// journals.openedition.org/edl/1318; DOI : https://doi.org/10.4000/edl.1318 


\section{AYMON DE MONTFALCON ET L'OBSERVANCE: LA FONDATION CONTROVERSÉE DES COUVENTS DE SAVIGNY, DE SAINTE-CATHERINE DU JORAT ET DE MORGES}

Attaché à l'Observance, tendance acquise vraisemblablement au contact de sa région d'origine, la Savoie, Aymon de Montfalcon va entreprendre la fondation de deux couvents dans le Pays de Vaud durant son épiscopat: les carmes de Sainte-Catherine du Jorat et les franciscains observants de Morges. Il mena à son terme également un projet de fondation laissé en suspens par son prédécesseur, l'établissement du Tiers-Ordre franciscain de Savigny. Bien qu'appartenant tous trois à des ordres différents, ils ont néanmoins comme point commun d'être issus de la mouvance observante.

1. Situation des couvents mendiants en Savoie et en Pays de Vaud au $X V^{e}$ siècle

À l'entrée en fonction d'Aymon de Montfalcon en tant qu'évêque de Lausanne en mai 1491, le Pays de Vaud compte huit couvents considérés comme faisant partie des ordres mendiants ${ }^{1}$. Leur fondation correspond à deux vagues bien distinctes. La première prend place au XIII ${ }^{\mathrm{e}}$ siècle, lors de la création de ces ordres. Sont issus de cette période le couvent dominicain de Lausanne, ainsi que les trois couvents franciscains de

I. L'établissement des ermites de Saint-Augustin de Vevey, fondé entre 1297 et 1301, a été supprimé en 1312. Il ne peut donc pas être comptabilisé parmi les couvents existants dans la fourchette de temps étudiée. S. Vocanson-Manzi, Les ordres mendiants dans le Pays de Vaud (XIII ${ }^{e}-X V I^{e}$ siècle), thèse en cours à l'Université de Lausanne sous la dir. du Prof. B. Andenmatten. 
Lausanne, Grandson et Nyon ${ }^{2}$. Après une interruption de plus d'un siècle, au cours de laquelle aucune nouvelle communauté mendiante ne s'établit dans le Pays de Vaud, la seconde vague de fondations se situe au $\mathrm{XV}^{\mathrm{e}}$ siècle et s'inscrit dans le contexte de la réforme observante. Cette dernière correspond à une volonté, de la part d'une frange des frères, de revenir aux valeurs primitives qui avaient formé le terreau de la création de leurs ordres et donc de retourner à une observance stricte de leur règle. Les promulgateurs de cette réforme pointaient notamment du doigt les nombreux privilèges accordés aux différents ordres par la papauté, leur ouvrant la possibilité de pratiquer la pauvreté au quotidien de manière plus souple. Le système de rentes mais également de provisions et de greniers, entre autres, apparaissait, aux yeux de ces derniers, comme allant totalement à l'encontre des préceptes désirés et pensés par les fondateurs. À la suite de ces mouvements de réformes, apparaissent donc de nouveaux couvents dans l'ensemble du territoire européen, dont trois dans le Pays de Vaud: les clarisses réformées (colettines) de Vevey et $\mathrm{d}^{\prime} O \mathrm{rbe}^{3}$, ainsi que les dominicains observants de Coppet ${ }^{4}$.

Dans le contexte plus large de la principauté savoyarde, les mendiants étaient présents avec une dizaine de couvents depuis le XIII siècle 5 . L'Observance va leur donner l'occasion de densifier de manière conséquente leur réseau d'établissements ${ }^{6}$ et d'arriver à quasiment en doubler le nombre en un siècle 7 . La caractéristique principale des couvents observants, savoyards et vaudois, est qu'ils correspondent tous à de nouvelles fondations, aucun établissement antérieur de ces régions n'ayant accepté le passage à l'Observance ${ }^{8}$. Les couvents vaudois du XIII ${ }^{\mathrm{e}}$ siècle sont donc demeurés dans la mouvance conventuelle jusqu’à leur suppression, lors du passage à la Réforme de ces régions, entre 1536 et 1555 .

2. La fondation de ces quatre couvents a lieu respectivement en 1234,1258 , 1289-1298 et 1295-1296.

3. Vevey, 1422-1424; Orbe, 1426-1430.

4. Fondation en 1490.

5. Selon les calculs de J.-P. Leguay, la Savoie compte en 1500 un total de 21 couvents («Urbanisme et ordres mendiants», p. 171).

6. Plus particulièrement pour les frères mineurs. Cf. É. Pierregrosse, «Foyers et diffusion de l'Observance dans les domaines de la Maison de Savoie (Piémont exclu) aux XVe-XVI" siècles", p. 255.

7. J.-P. Leguay, «Urbanisme et ordres mendiants», p. 169.

8. É. Pierregrosse, «Foyers et diffusion de l'Observance dans les domaines de la Maison de Savoie (Piémont exclu) aux XVe-XVIe siècles», p. 256. 
Au niveau de la chronologie, le $\mathrm{XV}^{\mathrm{e}}$ siècle amène des fondations de manière relativement régulière en Savoie avec deux vagues importantes, l'une entre 1413 et 1430 environ ${ }^{9}$, et l'autre entre 1451 et $1481^{10}$. Les établissements fondés par Aymon de Montfalcon dans le Pays de Vaud à la fin du $X^{e}$ siècle se situent donc en droite ligne de ceux que nous venons de mentionner pour la Savoie, avec comme maître-mot l'Observance.

\section{Les fondations observantes d'Aymon de Montfalcon}

Comme nous venons de le voir, aucun établissement antérieur au $\mathrm{XV}^{\mathrm{e}}$ siècle n'a, dans le Pays de Vaud comme en Savoie, accepté le passage à l'Observance. Par conséquent, si Aymon de Montfalcon avait pour ambition d'implanter l'Observance en terre vaudoise, il lui fallait d'emblée imaginer la fondation de nouveaux établissements. Ancien proche de la cour de Savoie, il est tout à fait attendu que si volonté de fonder des couvents il y avait, ces derniers seraient observants; et c'est ce qui se produisit. Selon l'ensemble de la documentation à notre disposition, il semblerait que l'évêque ait attendu environ trois ans après son arrivée dans sa nouvelle fonction lausannoise avant d'entamer des démarches dans ce sens. Trois couvents mendiants sont construits dans les premières années de son épiscopat. Deux sont exclusivement de son fait: les franciscains colétans de Morges et les carmes de Sainte-Catherine du Jorat. Quant au troisième, le couvent du Tiers-Ordre franciscain de Savigny, les tractations en vue de sa fondation avaient commencé avant l'arrivée du nouvel évêque. Elles s'étaient cependant brutalement

9. Avec les fondations de dominicains à Chambéry en 1413, à Annecy en 1422, de carmes à Pont-de-Beauvoisin en 1419, de clarisses colettines à Vevey et Orbe en 1422 et 1424-1426 et d'ermites de Saint-Augustin à Thonon en 1427. Cf. notamment J.-P. Leguay, «Urbanisme et ordres mendiants», p. 170.

Io. Avec les fondations de franciscains observants à Belley en 1451, à Myans en 1458, à Nice en 1460, à Chambéry en 1462, à Cluses en 1471, à Saint-Michel-de-Moûtier en 1471, à Pont-de-Vaux en 1472, à Sospel en 1480 et à Bourg-en-Bresse en 1481; pour les clarisses, il y a les fondations à Chambéry en 1471 et à Genève en 1474-1476; nous trouvons encore la fondation d'un établissement d'ermites de Saint-Augustin au Pont-d'Arve (Genève) en 1480. Cf. notamment É. Pierregrosse, «Foyers et diffusion de l'Observance dans les domaines de la Maison de Savoie (Piémont exclu) aux XVe XVI siècles», p. 260 et J.-P. Leguay, "Urbanisme et ordres mendiants», p. 170. 
interrompues quelques jours seulement après les premières démarches, suite au décès de Benoît de Montferrand, prédécesseur d'Aymon de Montfalcon. Cet établissement n'était par ailleurs pas issu de la volonté de l'ancien évêque, mais d'une requête de Pierre de Roseto, vicaire de la province de Bourgogne et de Strasbourg du Tiers-Ordre franciscain. Aymon de Montfalcon n'a donc fait que reprendre le projet et l'a mené à son terme. Nous nous concentrerons par conséquent dans cette étude sur les deux fondations de Morges et du Jorat, tout en incluant de manière sporadique celle de Savigny.

\subsection{Un choix: des mendiants observants}

Le point commun évident entre les trois fondations mentionnées ci-dessus est l'Observance, bien que toutes trois ne correspondent pas à une même manière de la pratiquer et de la penser. Or, comme nous venons de le voir, Aymon de Montfalcon, de par son origine et ses relations, a forcément été influencé par les couvents observants de Savoie. La littérature secondaire, et notamment Jacques Fodéré ${ }^{11}$ qui écrit au XVII ${ }^{\mathrm{e}}$ siècle, se plaît à voir un lien personnel entre Annable d'Antioche, premier gardien des frères mineurs de Morges, et l'évêque de Lausanne. En effet, ce prédicateur franciscain, reconnu pour ses talents d'orateur, était lié à la cour de Savoie qu'il avait rejointe à la suite de la duchesse Anne de Chypre ${ }^{12}$. Le prélat lausannois aurait donc fait venir sur ses terres le frère Annable qu'il avait déjà entendu prêcher, celui-ci étant un prédicateur qui s'était apparemment déjà rendu dans la région. Le but de l'évêque était cependant de faire rester Annable afin que lui et ses sujets puissent bénéficier de ses prédications ${ }^{13}$. Le prédicateur était

II. J. Fodéré, Narration Historique et Topographique de Couvents de l'ordre de $S$. François et Monastères de S. Claire érigez en la Province anciennement appelée de Bourgogne, à présent S. Bonaventure, p. 839-842.

I2. B. Andenmatten, "Antioche, Annable d'".

I3. "[...] il frequentoit souvent ce beau et aggreable pays de Vaux, separé de celuy de Chablais par le lac de Geneve, le tout estant des Estats du Duc de Savoye, où pour ses predications il edifioit grandement le peuple, parmy lequel il acqueroit une belle reputation, par sa bonne vie, particulierement R. Aymond de Montfalcon Evesque de Lausanne l'avoit en une estroicte affection" (J. Fodéré, Narration Historique et Topographique de Couvents de l'ordre de S. François et Monastères de S. Claire érigez en la Province anciennement appelée de Bourgogne, à présent S. Bonaventure, p. 839, repris 
déjà religieux au couvent de Cluses, lui-même fondé, entre autres, par une figure importante de l'Observance, le frère Jean Bourgeois ${ }^{14}$. Cette fondation morgienne se situe donc en droite ligne des couvents savoyards observants précédemment mentionnés. Cette filiation peut tout d'abord se voir dans le choix de l'ordre: des franciscains colétans, issus de la réforme de Colette de Corbie qui a notamment été à l'origine des couvents féminins réformés de Vevey et d'Orbe, mais également de nombreux autres en Savoie. Toutes ces fondations ont par ailleurs été soutenues par la Maison de Savoie. Ensuite, par la volonté de placer à sa tête le personnage d'Annable d'Antioche, lui aussi sorti des rangs de l'Observance savoyarde. Quant au choix du lieu, l'évêque avait décidé, dans un premier temps, d'implanter le couvent à Lausanne. Or, comme nous le savons, cette ville possédait déjà un couvent influent qui entretenait de bons rapports avec le Conseil de ville et qui, par ailleurs, avait rejeté à deux reprises le passage à l'Observance ${ }^{15}$. Il était donc difficile de justifier l'implantation d'un nouveau couvent concurrent, tant au niveau de la doctrine que du partage de la zone terminaire et donc des quêtes et des dons. Par ailleurs, il semble que les habitants de Morges aient formulé expressément la requête d'avoir l'établissement dans leur ville. Pour cela, ils se sont adressés au duc Philibert de Savoie dont dépendait la ville; ce dernier, par une lettre adressée à Aymon de Montfalcon et datée du 20 juillet 1497, demanda à ce que les frères mineurs s'installent dans cette localité ${ }^{16}$. Il s'agit donc d'une implantation urbaine d'un couvent appartenant à une mouvance observante.

par A. Wildermann, «Morges», p. 400 et M.-P. Anglade, "Les cordeliers de Morges (1497-1536)», p. 141).

I4. J.-P. Leguay, «Urbanisme et ordres mendiants», p. 170; É. Pierregrosse, «Foyers et diffusion de l'Observance dans les domaines de la Maison de Savoie (Piémont exclu) aux XVe-XVI ${ }^{\mathrm{e}}$ siècles", p. 258.

I5. S. Manzi, "Les couvents mendiants et les autorités laïques de Lausanne", p. $125-127$.

i6. Selon J. Fodéré, "on [les habitants de Morges et le duc] luy representa que dans sa Cité de Lausanne, y avoit desja un beau Convent des PP. Conventuels, du mesme Ordre S. François, \& qu'il seroit plus utile pour la commodité de ses Diocésains, de le faire autre part, mesmement en la ville de Morges, attendu le zele des habitans \& la volonté du Prince» (J. Fodéré, Narration Historique et Topographique de Couvents de l'ordre de S. François et Monastères de S. Claire érigez en la Province anciennement appelée de Bourgogne, à présent S. Bonaventure, p. 840). 
Concernant les carmes du Jorat, la démarche fut quelque peu différente. Il semblerait qu'Aymon de Montfalcon ait convoqué, à l'Avent 1494, le frère Henri Riond afin de lui faire part de son projet de fondation ${ }^{17}$. Ce frère s'est ensuite rendu aux États de Vaud, tenus à Moudon en février de l'année suivante, pour exposer le projet de fondation de l'évêque d'une institution à l'emplacement de l'ancien hôpital à demi-ruiné de Sainte-Catherine, dans les bois du Jorat ${ }^{18}$. Le rattachement au courant de l'Observance de cet établissement se fait donc selon un biais tout à fait différent de celui de Morges. Le lieu choisi pour son emplacement détermine sa nature: il appartient à ce que l'on nomme communément les fondations «du désert» ou «désertiques». En effet, elles ont comme caractéristique de se trouver dans une zone non urbaine, périphérique, éloignée du centre des localités, en l'occurrence de la ville de Lausanne, et surtout proche, voire à l'intérieur, d'une forêt, ici le Grand bois du Jorat. La forêt est justement perçue, dans l'imaginaire médiéval chrétien, comme apparentée au désert biblique dans lequel $s^{\prime}$ installe l'ermite; celui-ci est alors à la marge entre nature et culture ${ }^{19}$. Le Jorat doit donc être considéré comme un "désert-forêt» ${ }^{20}$, dans lequel vivent, toujours selon l'imaginaire médiéval, aussi bien des voleurs, des brigands que des assassins, contraints de se cacher dans un monde en marge, que des âmes chrétiennes venues chercher la quiétude pour la prière $^{21}$. Le choix de ce bâtiment préexistant, l'hôpital Sainte-Catherine, pour accueillir le nouveau couvent n'est donc pas anodin: situé sur la route reliant Lausanne à Moudon, Fribourg et Berne ${ }^{22}$, il permettra, selon la volonté de l'évêque, de favoriser le passage du Jorat ${ }^{23}$ qui, pour les Lausannois du Moyen Âge, représentait un lieu dangereux, peuplé de brigands et d'étrangers ${ }^{24}$. Lordre des carmes a par ailleurs lui aussi

17. AVL, Corps de Ville, A 183 et A 183 bis, 5 février 1495. Je profite de cette occasion pour remercier chaleureusement Lionel Dorthe qui m'a permis d'élucider toutes les subtilités de ce document.

18. AVL, Corps de Ville, A 183 bis.

I9. J. Le Goff, «Le désert-forêt dans l'Occident médiéval», p. 74; L. Dorthe, «Les brigands du Jorat aux marges de Lavaux, entre imaginaire et réalité», p. 123.

2o. Ibid., p. 123.

2I. Ibid., p. 124.

22. F. Huot, "Couvent (Ste-Catherine) du Jorat (Lausanne VD)», p. 1170.

23. AVL, Corps de Ville, A 183 bis.

24. L. Dorthe, «Les brigands du Jorat aux marges de Lavaux, entre imaginaire et réalité», p. 124 sq. 
connu des mouvements apparentés à l'Observance durant le $\mathrm{XV}^{\mathrm{e}}$ siècle. Preuve en est l'affiliation en 1432 de l'unique autre couvent de cet ordre en Suisse, à Géronde en Valais, au mouvement de réforme initié par Thomas Connecte en Italie, nommé la Congrégation de Mantoue ${ }^{25}$. Cet établissement sera par la suite, en 1489 , réintégré à la province de Narbonne ${ }^{26}$ à laquelle sera également affiliée Sainte-Catherine du Jorat. Au-delà du lieu choisi pour son emplacement qui, comme nous l'avons $\mathrm{vu}$, le rattache au mouvement de l'Observance par le biais des fondations dites du désert, le couvent du Jorat pourrait donc avoir été affilié à l'Observance carme de par son rattachement à la province de Narbonne, puisque la réforme, menée par Jean Soreth, se propagea en France à partir des années $1460^{27}$. Cependant, aucune source ne vient attester précisément cette filiation.

Quant au couvent du Tiers-Ordre franciscain de Savigny, bien que n'ayant pas été à proprement parler désiré par l'évêque, il appartient, lui aussi à l'Observance. Tout d'abord de par son emplacement. Tout comme celui de Sainte-Catherine, le couvent du Tiers-Ordre se situe dans la zone du nord de Lausanne, touchant le Jorat, et il s'installe sur les bases d'une ancienne chapelle préexistante ${ }^{28}$. L'établissement a été fondé dans un bourg abandonné depuis quelques temps par ses habitants, partis vivre plus au sud dans la région de Lutry, au bord du lac ${ }^{29}$. Par ailleurs, les informations que nous possédons au sujet de son fondateur, Pierre de Roseto, laissent à penser qu'il appartient de plein droit au courant observant. Celui-ci était frère dans un couvent franciscain de l'Observance avant d'entrer, à une date indéterminée, dans le Tiers-Ordre ${ }^{30}$. Fort de ces considérations, nous pourrions envisager de voir le problème à l'envers. La fondation de Savigny datant de mai 1491, elle est par conséquent

25. Thomas Connecte n'est pas seulement l'instigateur de la Congrégation de Mantoue, mais également le fondateur du couvent de Géronde. F. Huot, «Introduction générale», p. 1134 et "Couvent (Notre-Dame) de Géronde», p. 1138 sq.; P. W. Janssen, Les origines de la réforme des carmes en France au XVII siècle, p. 26; M. de SainteMarie, "Carmel (Ordre de Notre-Dame du Mont-Carmel)", cols 1084 sq.; L. Saggi, "Carmelitani», cols 462 sq.

26. F. Huot, «Introduction générale», p. 1134 et "Couvent (Notre-Dame) de Géronde», p. 1138 sq.

27. P. W. Janssen, Les origines de la réforme des carmes en France au XVII e siècle, p. 28.

28. A. Wildermann, «Savigny», p. 730.

29. Ibid.

30. J.-P. Bastian, «Réforme observante et emprise territoriale», p. 215. 
antérieure à celle de Sainte-Catherine. Elle a donc pu être un modèle pour l'évêque qui, trois ans plus tard, décida d'installer d'autres frères, non loin de là, dans un couvent qui, cette fois, lui sera entièrement attribué.

\subsection{Les premières pierres et les donations}

Au-delà de la volonté de fondation, il fallait donner aux frères les moyens concrets et financiers de construire ces couvents. C'est bien évidemment Aymon de Montfalcon qui, dans les deux cas, est le principal donateur. Le prélat donna un pré aux franciscains de Morges, entre la fin du mois d'août et le début du mois de septembre 1497, terrain qu'il avait préalablement acheté en vue de la construction de l'institution, juste après avoir accepté de l'établir dans cette ville ${ }^{31}$. Les carmes reçurent quant à eux 400 toises de terrain autour de la ruine de l'église de SainteCatherine, ainsi que l'affouage, l'usage du bois de construction et les droits de pâturage dans le reste de la forêt du Jorat ${ }^{32}$. En revanche, dans un cas comme dans l'autre, des conflits retardèrent la pose de la première pierre de l'église. À Morges, alors que le duc de Savoie et les habitants avaient eux-mêmes réclamé l'installation des frères, c'est avec le curé de la localité, qui craignait d'être lésé par l'arrivée d'une concurrence mendiante, que les franciscains durent composer. Le conflit fut réglé en août 1498 par l'évêque lui-même qui proposa en dédommagement au prêtre une somme de 100 florins pour l'acquisition d'une rente de 5 florins annuels ${ }^{33}$. Le geste symbolique de la pose intervint donc le 24 mai 1500 , accompagné d'une donation de 4000 florins en vue de la construction de l'édifice ${ }^{34}$. D'autres donateurs principaux concoururent financièrement à la construction du couvent de Morges. En premier lieu, nous retrouvons

3I. J. Fodéré, Narration Historique et Topographique de Couvents de l'ordre de S. François et Monastères de S. Claire érigez en la Province anciennement appelée de Bourgogne, à présent S. Bonaventure, p. 840 sq.; M.-P. Anglade, "Les cordeliers de Morges (1497-1536)», p. 142 sq.

32. AVL, Corps de Ville, A 186 et 187; Extraits des manuaux du Conseil de Lausanne (1383-1511), p. 114; F. Huot, "Couvent (Ste-Catherine) du Jorat (Lausanne VD)", p. $1170 \mathrm{sq}$.

33. Archives cantonales vaudoises (ACV), Minutaire Perceval Gruet, Dg 133, p. 67 sq.; A. Wildermann, «Morges», p. 401.

34. ACV, Minutaire Perceval Gruet, Dg 133, p. 93; M.-P. Anglade, «Les cordeliers de Morges (1497-1536)», p. 144; A. Wildermann, «Morges», p. 401. 
bien évidemment Philibert, duc de Savoie, qui s'était personnellement impliqué dans la requête du choix d'emplacement de l'établissement. Il fit une donation annuelle de 100 florins d'or jusqu'à l'achèvement de la construction, que sa femme Marguerite d'Autriche continua à verser après sa mort survenue en $1504^{35}$. Lautre donateur important fut le frère d'Annable d'Antioche, Georges, qui offrit 100 florins d'Allemagne ${ }^{36}$.

Les carmes durent s'armer de davantage de patience pour obtenir la première pierre de leur église. En effet, les relations étaient déjà très tendues entre l'évêque et les habitants de Lausanne du temps du prédécesseur d'Aymon, Benoît de Montferrand ${ }^{37}$. Or, il se trouve qu'une partie du Jorat, et en particulier le lieu choisi par l'évêque pour l'installation des frères carmes, "appartenait de plein droit à la commune de Lausanne " ${ }^{38}$. Les conseillers de ville s'élevèrent donc contre la première donation de terrains qu'Aymon avait faite en faveur du couvent ${ }^{39}$, ce qui retarda fortement le début de la construction ${ }^{40}$. En août 1510 intervint une modification de la première donation d'Aymon en faveur des carmes, afin de contenter les bourgeois de Lausanne. Les 400 toises se réduisirent à un enclos fermé et délimité. Pour le reste, les frères n'obtinrent que la première herbe sur les pâturages ${ }^{41}$. Les nouvelles conditions furent

35. J. Fodéré, Narration Historique et Topographique de Couvents de l'ordre de $S$. François et Monastères de S. Claire érigez en la Province anciennement appelée de Bourgogne, à présent S. Bonaventure, p. 841.

36. Ibid.

37. C'est notamment durant cet épiscopat que la Ville inférieure et la Cité ont décidé de fusionner pour ne constituer plus qu'une seule communauté politique face au pouvoir du prince-évêque. Cf. à ce sujet C. Thévenaz Modestin, Un mariage contesté.

38. F. Huot, "Couvent (Ste-Catherine) du Jorat (Lausanne VD)», p. 1171.

39. Dans les extraits des procès-verbaux du Conseil de ville publiés par E. Chavannes, nous retrouvons le refus catégorique de ce dernier d'admettre la nouvelle fondation. En date du 17 août 1497, il est ainsi indiqué que: Prior cartusie nove (sic) que erigitur in hospitali beate Katharine presentavit litteras sue fondacionis et requisivit ven. consilium quod dignaretur laudare et approbare dictam fondacionem. Qua visa, predictum ven. consilium, quia locus et circonstancie dicte fondacionis pertinent pleno jure communitati Lausanne, venerabile. consilium ipsam fondacionem non admisit, in Extraits des manuaux du Conseil de Lausanne (1383-1511), p. 111 ; F. Huot, "Couvent (Ste-Catherine) du Jorat (Lausanne VD)», p. 1171.

40. Ibid.

4I. Archives de la ville de Lausanne (AVL), Corps de Ville, K 2; Extraits des manuaux $d u$ Conseil de Lausanne (1383-1511), p. 115; F. Huot, "Couvent (Ste-Catherine) du Jorat (Lausanne VD)», p. 1171. 
définitivement acceptées entre septembre 1511 et août 1512 par la ville et les frères ${ }^{42}$. La construction de l'église ne semble pourtant pas être intervenue avant une décennie. Preuve en est l'existence d'un compte spécifique pro edificatione ecclesie conventus Sante Catherine in Joreto ${ }^{43}$ datant des années 1522 à $1531^{44}$. C'est le neveu d'Aymon, Sébastien de Montfalcon, monté sur le siège épiscopal de Lausanne en 1517, qui rassembla les fonds nécessaires à l'ouvrage ${ }^{45}$. Quant aux bâtiments conventuels, ils avaient dû être habitables bien avant cette date. En effet, au moins une partie de l'ancien hôpital aurait servi aux frères et avait subi des réparations avec notamment le concours financier du Conseil de ville de Lausanne qui, en 1498-1499, offrit 4000 tuiles ${ }^{46}$. Aucune donation pécuniaire ne semble avoir été faite par Aymon aux carmes, comme cela avait été le cas en 1500 pour les franciscains de Morges. Cependant, deux nouvelles donations intervinrent peu avant sa mort en 1517, constituées pour l'essentiel de poses de vignes ${ }^{47}$. D'autres donateurs se manifestèrent pour l'édification de l'établissement, notamment Charlotte de Vergy, veuve de Jean d'Estavayer, bailli de Vaud, en $1514^{48}$.

Le cas de Savigny est à traiter complètement à part. Bien que n'étant pas issu de la volonté d'Aymon de Montfalcon, l'évêque en a pourtant facilité l'implantation. En juillet 1491, soit moins de trois mois après l'autorisation de Benoît de Montferrand, décédé entre temps, le prélat lausannois nouvellement élu accorda une indulgence de quarante jours aux fidèles qui se rendraient à Savigny pour y visiter la chapelle ${ }^{49}$. Il

42. AVL, Corps de Ville, K 3, K 4, K 5; Extraits des manuaux du Conseil de Lausanne (1383-1511), p. 115 ; F. Huot, "Couvent (Ste-Catherine) du Jorat (Lausanne VD)", p. 1171.

43. ACV, C VI k 1, comptes pour l'édification du couvent de Sainte-Catherine, 1522-1531.

44. M. Grandjean, La ville de Lausanne, p. 314.

45. AVL, Corps de Ville, C VI k 1; M. Grandjean, La ville de Lausanne, p. 314 sq.;

F. Huot, "Couvent (Ste-Catherine) du Jorat (Lausanne VD)», p. 1171.

46. AVL, D 221, comptes 1498-1499, fol. 24; M. Grandjean, La ville de Lausanne, p. 314.

47. Extraits des manuaux du Conseil de Lausanne (1383-1511), p. 115; F. Huot, "Couvent (Ste-Catherine) du Jorat (Lausanne VD)», p. 1171.

48. ACV, D 1 43, I, le 17 mai 1514; M. Grandjean, Lausanne, p. 400.

49. Archives communales de Lutry, Bleu F 27, Registrum, fol. 87-88v; A. Wildermann, "Savigny", p. 730 ; J.-P. Bastian, "Réforme observante et emprise territoriale», p. 213 sq. 
renouvela son soutien afin de recueillir des fonds en vue de la réparation et de la construction des bâtiments conventuels à diverses reprises, en tout cas jusqu'en $1494^{50}$. Même les conflits, qui avaient occasionné un procès retentissant tenu à Bâle et opposant les religieux de Savigny à l'évêque de Lausanne, mettent en lumière le soutien apporté par les deux prélats, d'abord Benoît de Montferrand, puis Aymon de Montfalcon, aux frères du Tiers-Ordre dans leur installation ${ }^{51}$. En effet, l'évêque avait donné une chapelle au groupe de frères, outrepassant ainsi les droits sur les paroisses lausannoises qui appartenaient alors au chapitre ${ }^{52}$. C'est bien ce dernier qui était impliqué pleinement dans le procès ${ }^{53}$. Donation il y a donc bien eu de la part du prélat, même si celle-ci a porté à controverse.

\section{Conclusion}

Nous avons donc affaire ici à trois fondations mendiantes datant des années 1490-1500, dans le Pays de Vaud, à proximité de Lausanne et sorties de terre grâce au concours de l'évêque fraîchement élu: Aymon de Montfalcon, prélat soucieux de l'orthodoxie puisqu'il choisit la mouvance observante. Le prélat arrive en Pays de Vaud depuis la Savoie avec un modèle en tête: les couvents mendiants de l'Observance, et plus particulièrement celle mise en place par Colette de Corbie dans la première moitié du siècle. Or, il se trouve que, tout comme en Savoie, le Pays de Vaud ne connaît pas de passage à l'Observance pour les couvents "primitifs", fondés au XIII siècle. Les deux tentatives avortées de passage à l'Observance, notamment par le concile de Bâle, des franciscains conventuels de Lausanne en sont un bon exemple. En effet, dans les comptes des prieurs de la Ville inférieure de Lausanne, en date du mercredi 23 septembre 1439, nous trouvons des traces du paiement de repas pour des conseillers "qui donnèrent réponse à Maître François au sujet de la réformation prédite en la maison desdits religieux", soit les franciscains de Lausanne. La seconde mention, plus allusive, date d'octobre 1440 et concerne une rencontre entre un certain Nicod Festi et

50. A. Wildermann, «Savigny», p. 730 sq.

5I. J.-P. Bastian, «Réforme observante et emprise territoriale», p. 222.

52. Ibid., p. 213.

53. Ibid., p. 223. 
les conseillers de ville à propos d'une requête des frères mineurs. Le refus de passage à l'Observance a donc été soutenu par la ville, par le biais de ses conseillers, qui, du moins dans le premier cas, avait fourni le lieu de la rencontre et payé une partie des repas ${ }^{54}$.

La volonté de l'évêque d'établir dans un premier temps son nouveau couvent à Lausanne semble quant à elle motivée par une volonté d'asseoir son autorité. La décision de fonder un établissement mendiant réformé qui lui soit directement affilié, dans une ville qui en possédait déjà un resté fidèle au mouvement conventuel, en est un premier indice. Le fait que ce premier couvent entretienne des rapports étroits avec le Conseil de ville, avec lequel l'évêque est justement en conflit, n'est non plus pas anodin. Enfin, décider de fonder un second couvent, celui de Sainte-Catherine du Jorat, en empiétant sur les terres appartenant à cette même entité politique, le Conseil de ville, n'est peut-être pas innocent non plus et démontre à nouveau une volonté d'affirmer sa puissance et ses prérogatives. En définitive, le couvent franciscain se fera à Morges et les terrains cédés aux carmes réduits au strict minimum.

Le cas de Savigny semble également intéressant puisqu'il oppose cette fois-ci l'évêque au chapitre par le biais de la donation d'une chapelle qui relevait en réalité de l'autorité capitulaire. Par ces trois fondations mendiantes dont il a soutenu l'installation, nous percevons l'image d'un évêque qui tente, sans grand succès, d'imposer ses prérogatives aussi bien à la ville qu'au chapitre, après l'épiscopat compliqué de Benoît de Montferrand. Souvenons-nous, par ailleurs, que sa nomination en tant que prélat lausannois n'était pas le fait du chapitre, à qui revenait en principe le droit d'élection, mais résultait d'une intervention directe du duc de Savoie qui avait plaidé sa candidature à Rome. Ces tensions entre l'évêque de Lausanne d'un côté et la ville et le chapitre de l'autre se ressentent donc également dans le cas des fondations conventuelles épiscopales. Son besoin d'asseoir son pouvoir à travers ces établissements ne va cependant pouvoir se concrétiser dans aucun des cas étudiés, que ce soit à Morges, dans le Jorat ou à Savigny.

Stéphanie Vocanson-Manzi

Université de Lausanne

54. S. Manzi, "Les couvents mendiants et les autorités laïques de Lausanne», p. $125-127$. 


\section{BIBLIOGRAPHIE}

\section{Sources}

Extraits des manuaux du Conseil de Lausanne (1383-1511), publ. et annotés par Ernest Chavannes, Lausanne, 1881, p. 3-378 (Mémoires et documents de la Société d'histoire de la Suisse romande, $1^{\mathrm{e}}$ série/XXXV).

Fodéré, Jacques, Narration Historique et Topographique de Couvents de l'ordre de S. François et Monastères de S. Claire érigez en la Province anciennement appelée de Bourgogne, à présent S. Bonaventure, Lyon, chez Rigaud, 1619.

\section{Travaux}

Andenmatten, Bernard, "Antioche, Annable d'", in Dictionnaire historique de la Suisse (en ligne: <http://www.hls-dhs-dss.ch/ textes/f/F12481.php>).

Anglade, Marie-Pascal, "Les cordeliers de Morges (1497-1536)", Revue historique vaudoise, 22 (1914), p. 139-154.

Bastian, Jean-Pierre, "Réforme observante et emprise territoriale: l'éphémère implantation du couvent du Tiers-Ordre de SaintFrançois à Savigny, diocèse de Lausanne, 1491-1531", Revue historique vaudoise, 125 (2017), p. 201-236.

Dorthe, Lionel, "Les brigands du Jorat aux marges de Lavaux, entre imaginaire et réalité", in La mémoire de Lavaux. Territoire, population, éducation, société, éd. par Jean-Pierre Bastian, Bière, Cabedita, 2014, p. 107-134.

Grandjean, Marcel, La ville de Lausanne. Introduction, extension urbaine, ponts, fontaines, édifices religieux (sans la cathédrale), hospitaliers, édifices publics, Bâle, Birkhaüser, 1965 (Les Monuments d'art et d'histoire du canton de Vaud, 1). 
-, Lausanne. Villages, hameaux et maisons de l'ancienne campagne lausannoise, Bâle, Birkhaüser, 1981 (Les Monuments d'art et d'histoire du canton de Vaud, 4).

Нuот, François, "Introduction générale», in Helvetia Sacra, VI. Die Karmeliter in der Schweiz, bearbeitet von D. François Huot, redigiert von Brigitte Degler-Spengler, Bern, Francke, 1974, p. 11331136.

—, "Couvent (Notre-Dame) de Géronde», in Helvetia Sacra, VI. Die Karmeliter in der Schweiz, bearbeitet von D. François Huot, redigiert von Brigitte Degler-Spengler, Bern, Francke, 1974, p. 1137-1169.

—, "Couvent (Ste-Catherine) du Jorat (Lausanne VD)", in Helvetia Sacra, VI. Die Karmeliter in der Schweiz, bearbeitet von D. François Huot, redigiert von Brigitte Degler-Spengler, Bern, Francke, 1974, p. 1170-1175.

Janssen, Petrus Wilhelmus, Les origines de la réforme des carmes en France au XVII siècle, La Haye, M. Nijhoff, 1963.

Le Goff, Jacques, "Le désert-forêt dans l'Occident médiéval", in L'imaginaire médiéval, Paris, Gallimard, 1985, p. 59-75.

Leguay, Jean-Pierre, "Urbanisme et ordres mendiants. L'exemple de la Savoie et de Genève (XIII ${ }^{\mathrm{e}}$-début XVI ${ }^{\mathrm{e}}$ siècle)", in Religion et mentalités au Moyen Âge: mélanges en l'honneur d'Hervé Martin, éd. par Sophie Cassagnes-Brouquet, Amaury Chauou, Daniel Pichot, Lionel Rousselot, Rennes, Presses universitaires de Rennes, 2003, p. 167-182.

Manzi, Stéphanie, "Les couvents mendiants et les autorités laïques de Lausanne: des tentatives observantes à la sécularisation protestante (1436-1536)", in Religion et pouvoir. Citoyenneté, ordre social et discipline morale dans les villes de l'espace suisse (XIV XVIII ${ }^{e}$ siècles), éd. par Mathieu César, Marco Schmidt, Neuchâtel, Alphil, 2014, p. 117-136.

Pierregrosse, Édith, "Foyers et diffusion de l'Observance dans les domaines de la Maison de Savoie (Piémont exclu) aux XV $X_{-}$ $\mathrm{XVI}$ siècles", in Identités franciscaines à l'âge des réformes, éd. par Frédéric Meyer, Ludovic Viallet, Clermont-Ferrand, Presses universitaires Blaise Pascal, 2005, p. 255-273. 
SAGGI, Ludovico, "Carmelitani", in Dizionario degli istituti di perfezione, a cura di Guerrino Pelliccia, Giancarlo Rocca, Rome, Edizioni Paoline, 1974, t. 2, cols 460-476.

Sainte-Marie, Melchior de, "Carmel (Ordre de Notre-Dame du MontCarmel)", in Dictionnaire d'histoire et de géographie ecclésiastiques, Paris, Letouzey et Ané, 1949, vol. 11, cols 1070-1104.

Thévenaz Modestin, Clémence, Un mariage contesté, Lausanne, Université de Lausanne, 2006 (Cahiers lausannois d'histoire médiévale, 38).

Wildermann, Ansgar, "Morges", in Helvetia Sacra, V/1. Die Franziskaner, die Klarissen und die regulierten FranziskanerTerziarinnen in der Schweiz. Die Minimen in der Schweiz, bearbeitet von mehreren Autoren, redigiert von Brigitte DeglerSpengler, Bern, Francke, 1978, p. 400.

—, "Savigny", in Helvetia Sacra, IX/2. Die Beginen und Begarden in der Schweiz, bearbeitet von mehreren Autoren, redigiert von Cecile Sommer-Ramer, Basel/Frankfurt am Main, Francke, 1995, p. $730-735$. 
